Résumés des conférences et travaux

\title{
Méthodes en histoire du monde portugais
}

\section{Méthodes en histoire du monde portugais}

Conférences de l'année 2012-2013

Dejanirah Silva-Couto

\section{OpenEdition \\ Journals}

\section{Electronic version}

URL: https://journals.openedition.org/ashp/1633

DOI: 10.4000/ashp.1633

ISSN: 1969-6310

\section{Publisher}

Publications de l'École Pratique des Hautes Études

\section{Printed version}

Date of publication: 1 September 2014

Number of pages: 290-297

ISSN: 0766-0677

\section{Electronic reference}

Dejanirah Silva-Couto, "Méthodes en histoire du monde portugais", Annuaire de l'École pratique des hautes études (EPHE), Section des sciences historiques et philologiques [Online], 145 | 2014, Online since 18 December 2014, connection on 06 July 2021. URL: http://journals.openedition.org/ashp/1633 DOI: https://doi.org/10.4000/ashp.1633 


\title{
MÉTHODES EN HISTOIRE DU MONDE PORTUGAIS
}

\author{
Maître de conférences : $\mathrm{M}^{\mathrm{me}}$ Dejanirah Silva-Couto
}

Programme de l'année 2012-2013 : I. Le Portugal et la Méditerranée orientale (2). - II. Le Portugal et l'Allemagne hitlérienne (3).

\section{Le Portugal et la Méditerranée orientale}

Dans le cadre du cycle triennal de conférences consacré à la politique méditerranéenne du Portugal ( $\mathrm{XV}^{\mathrm{e}}-\mathrm{XVI}^{\mathrm{e}}$ siècles), les travaux du séminaire 2012-2013 ont porté tout d'abord sur celle de D. Manuel (1499-1521). Le 12 novembre 1499, le souverain a répondu favorablement à l'appel du pape Alexandre VI enjoignant les princes de la Chrétienté à s'unir contre le Turc. Cependant, l'intervention lusitanienne, négociée à Rome par le cardinal D. Jorge da Costa, le seul membre portugais du Sacré Collège, fut subordonnée à deux conditions : le commandement de la coalition chrétienne serait confié à D. Manuel, qui pourrait percevoir les terços, autrement dit les revenus de la dîme écclesiastique affectés au financement des préparatifs militaires. L'initiative pontificale n'eut qu'un écho de pure forme, et D. Manuel, qui rappelons-le, n'avait jamais combattu personellement l'Islam, décida de passer en Afrique du Nord avec 6000 hommes. Ce fut là le début d'une politique typique du souverain, qui se proposa de conduire presque simultanément des interventions au Maroc (1499, 1501, 1503, $1508,1517)$ et des participations aux coalition anti-ottomanes $(1499,1501,1505,1516)$.

Ce projet explique la clause du contrat de mariage avec l'infante D. Maria, fille des Rois catholiques (rédigée le 12 avril 1500) sollicitant l'aide diplomatique de ses futurs beaux-parents dans la lutte contre le Turc. Cette clause s'inscrivait dans le contexte de la motion commune sur la Renovatio Ecclesiae (1498-1499), demandant la liberté de navigation pour les Portugais dans les eaux méditerranéennes dominées par la couronne d'Aragon. Cela étant, en 1500, la place du Portugal dans le dispositif pontifical était modeste : avec 8000 hommes, elle venait après l'Empire, les Rois catholiques (16 000) les États italiens (15000), la France et la Hongrie. Il ne devançait que le Danemark (6 000) Venise (2 000) et la Pologne (1 000). La contribution lusitanienne fut réevaluée à l'automne 1500 , peut-être en raison de l'engagement marocain : à l'instar de l'Angleterre, de l'Écosse et du Danemark elle serait désormais financière tandis que la France, l'Espagne et Venise étaient censées participer avec leurs forces navales; la Pologne et la Hongrie prêteraient leur concours avec des forces terrestres. Cependant, devant la réticence des Rois catholiques, peut désireux de soutenir financièrement l'effort de guerre, Venise, dans le contexte du conflit véneto-ottoman de 1499-1502, essaya d'impliquer davantage le Portugal. Elle envoya à Lisbonne son ambassadeur Domenico Pisani (suivi par son secrétaire Zuan Cretico), dans la foulée de la mission que avait conduit Pisani auprès des Rois catholiques. Pisani avait pour instruction de détourner D. Manuel de sa campagne nord-africaine (on préparait 
à Lisbonne l'expédition de Fez) et de lui proposer de rejoindre la flotte espagnole en Méditerranée. En réalité, les Rois avaient autre projet (soutenu par Louis XII de France) : celui de s'amparer du royaume de Naples (voir à ce propos le traité de Chambord du 10 octobre 1500, contresigné à Grenade le 25 novembre de la même année). Dans ce plan, on avait assigné à D. Manuel la jonction avec la flotte de D. Gonzalo Fernández de Cordoba et, incidemment, l'interception de la flotte ottomane dont on supposait qu'elle viendrait à la rescousse du roi de Naples.

La mission Pisani se solda d'abord par un échec : D. Manuel rappela son engagement en Afrique du Nord et les obstacles d'ordre économique, car les soldats, qui n'étaient pas payés pour la campagne d'Afrique, demanderaient des soldes pour aller au combat contre le Turc.

Contrairement à ce qui a été parfois affirmé, l'entourage de D. Manuel (en tout cas le parti « Bragance ») a été aussi reservé sur l'aventure nord-africaine que sur la croisade anti- ottomane. Il s'agissait d'abord de ne pas porter ombrage à la présence espagnole au Maghreb, en tenant compte de la rivalité luso-castillane pour la possession de Fez et des places-fortes de la côte méditerranéenne. D. Manuel avait pris un peu d'avantage sur ses beaux-parents en juin 1497 en obtenant du pape la bulle Ineffabilis et Summi qui donnait au Portugal le droit de conquête sur toutes les villes marocaines conquises, ce qui déplut fort aux Rois catholiques. Pour des raisons notamment économiques, le conseil royal fut également très reservé sur l'engagement dans la coalition anti-ottomane. Mais D. Manuel persista et annonça au pape (par lettre du 22 férvrier 1501), dans une démarche qui l'opposa à son conseil, la participation navale du Portugal. Moins impressionnante que celle de D. Fernando González de Cordoba (4 carraques génoises, 31 barges, 26 caravelles, 13 fustes, 7 galères légères, cent vingt pièces d'artillerie, 15000 hommes), la flotte de 1501, commandée par D. João de Meneses, comte de Tarouca, a réuni tout de même 30 voiles, dont 8 nefs et 22 caravelles. Les commandements ont été donnés à des capitaines de grande notoriété, tels que Tristão da Cunha (le découvreur éponyme de l'île), Pero de Anhaya (capitaine de l'armada du Mozambique en 1505 et premier capitaine de la forteresse de Sofala), Cid Barbudo, Gaspar Jusarte et quelques autres. Elle rassembla 3000 à 3500 hommes, moins que l'armée de Fez, évaluée à environ 26000 hommes.

Le séminaire s'est attaché ensuite à étudier ce qu'on a appelé conventionnellement « l'affaire de Mers-el-Kébir » (juillet 1501). On a ainsi constaté que la flotte du comte de Tarouca comportait une sous-escadre commandée par Miguel Corte Real, dont la mission consistait à s'amparer de Mers-el-Kébir et d'Oran (places-fortes que le traité de Tordesillas avait inclu dans la zone d'influence espagnole). D. Manuel chercha-t-il à réouvrir le comptoir d'Oran fermé en 1487, mentionné dans la conférence de l'année dernière ? Souhaita-t-il établir des bases à l'est de Melilla, de manière à assurer une présence portugaise en Méditerranée centrale, empiétant ainsi sur la zone d'influence des Espagnols? Ou désira-t-il faire d'Oran une base navale permettant de combattre la course ottomane ? On se souviendras que l'amiral Ottoman Kemal Re'îs captura en 1498 deux bâtiments corsaires portugais à Aboukir, une barge de 300 à 400 botte, puissament armée d'artillerie, avec un équipage de 180 hommes, et un petit galion. Quoi qu'il en soit, l'assaut contre Mers-el-Kébir, donné le 24 juillet 1501, échoua. D. João de Meneses se dirigea alors vers Cagliari, toucha Croton, Avlona et gagna Corfou le 29 septembre 1501 où il fit la jonction avec la flottte vénitienne. 
Il a semblé intéressant de relever les témoignages vénitiens sur l'armada portugaise de 1501. En effet, les Vénitiens furent impressionnés par les caractéristiques des bâtiments portugais : ceux-ci alliaient un faible tonnage à une extraordinaire puissance de feu. La majorité de la flotte était constituée par des navili piccolissimi «comme les navires d'Esclavonie sans hune (schiba) qui portent des vins à Venise », mais étaient dotés « d'un incroyable quantité d'artillerie, les plus petits ayant trois grosses bombardes de fer outre des petites pièces » (Mario Zen, provéditer de Nauplie, dans Marino Sanuto, I Diarii, vol. IV, p.166).

L'indiscipline des équipages portugais et les hésitations du commandement expliquent l'insuccès de D. João de Meneses. Des échauffourées éclatèrent à terre entre marins portugais et vénitiens, entraînant 80 morts du côté portugais. Des pillages ont été constatés. Entre-temps arriva la nouvelle du rappel de la flotte de Bayezit II, qui avait été envoyée contre Negropont. D. João de Menezes retourna au Portugal le 25 décembre 1501, sans avoir « détruit l'escadre ottomane en mer » comme D. Manuel lui avait ordonné, mais aussi après avoir refusé de participer aux opérations vénitiennes contre Durazzo et Santa Maura (Leucade) ou de se joindre à l'escadre de Philippe de Clèves qui navigait dans les eaux grecques. En réalité, cette expédition fut l'occasion pour la force navale portugaise d'attaquer les navires marchands italiens, génois et vénitiens qui faisaient du commerce avec les ports de l'Afrique du Nord, sous prétexte de la vente d'armes à feu aux maures d'Oran. Une carraque génoise, la Giustiniana, qui trafiquait entre l'Égypte et la Berbérie pour le compte de négociants musulmans fut arraisonnée de même qu'un brigantin génois. La cargaison de la Giustiniana fut confisquée temporairement et les musulmans qui se trouvaient à bord, emmenés captifs à Lisbonne (Biblioteca Nacional da Ajuda, Lisbonne, Ms.50-V-21, f. 25v). Le brigantin fut incorporé à la flotte portugaise. La course portugaise ne s'arrêta pas là : des galères en partance vers Oran, selon une route qui était censée éviter l'escadre de Kemal Re'îs, ont été arraisonnées par les Portugais dans la rade d'Almeria (lors du voyage de retour de la flotte de D. João). Ferdinand le Catholique s'est plaint auprès de D. Manuel de la violation des lois d'usage, qui interdisaient une opération de course dans un port d'une puissance amie. Or, pour éviter des incidents de ce genre, D. Manuel n'avait pas confié le commandement de l'armada portugaise à D. Diogo Fernandes de Almeida, prieur du Crato (le plus haut dignitaire de l'ordre de Saint-Jean de Jérusalem au Portugal). Vétéran de la course en mer Egée, élu en septembre 1502 capitaine des galions de la Religion, il remporta une victoire d'un certain retentissement contre le corsaire ottoman Kurtoglu en 1503 et retourna au Portugal en 1504. Il y a sens doute une relation à établir entre ce retour, les plans de D. Manuel d'asphyxie de l'Égypte qui prennent forme cette année-là, et les instructions en vue de la fondation de l'Estado da India dont fut chargé D. Francisco de Almeida, le frère de D. Diogo, en 1505.

En dépit d'une abondante littérature lusitanienne exaltant l'expédition de 1501 (voir l'Oratio de D. Pedro de Meneses dans Cataldi Epistolarum secunda pars) on constate que les gains politiques ont été modestes. D. Manuel n'a pas réussi à s'amparer de Mers-el-Kébir ou d'Oran, censés lui servir de monnaie d'échange dans le reéquilibrage des forces pour la possession d'enclaves sur la côte de Berbérie. Néanmoins, l'expédition servit les Rois catholiques, qui détournèrent D. Manuel, au moins pour quelque temps, de la conquête de Fez. 
Les travaux du second semestre de la conférence ont été consacrés plus précisément aux plans de D. Manuel concernant la croisade de Terre Sainte et à ses relations avec l'ordre de Saint-Jean de Jérusalem après l'échec de l'armada de 1501. L'auditoire du séminaire a donc initié la lecture et le commentaire d'une série de 21 documents inédits, dont la majorité est conservée dans les archives nationales de la Torre do Tombo à Lisbonne (AN / TT, Corpo Cronológico I et II et Núcleo Antigo 879) et à la Biblioteca Nacional de Portugal-BNP (Fundo Geral, cod.7638, f. 132v-133v). Ce corpus, assez homogène du point de vue chronologique (comportant des documents datés entre 1507 et 1517), permet notamment de dresser une biographie d'André do Amaral, vicechancelier de l'Ordre, et de mieux connaître la politique levantine de D. Manuel entre 1507 et 1512. On a ainsi commencé par souligner que D. Francisco de Almeida fut envoyé dans l'océan Indien en 1505, à fin d'occuper l'île de Soqotra et de construire une forteresse à l'entrée de la mer Rouge, tandis que le confesseur du roi, Frei Henrique de Coimbra (l'ancien chef de la mission franciscaine de 1500 à Calicut), fut envoyé à la cour d'Henri VII et à celle de Ferdinand d'Aragon (Isabelle étant décédée le 24 novembre 1504) dans le but de promouvoir la croisade de Terre Sainte, opération qui devait aboutir, in fine, au découpage du Proche-Orient entre trois alliés : le Portugal, l'Espagne et l'Angleterre. La tentative n'a pas eu de succès, car Ferdinand, en dépit de la crise de succession qui s'ouvrit en 1506, de sa rivalité avec Philippe le Beau et de son court exil à Naples, fit occuper définitivement Mers-el-Kébir en septembre 1505, Melilla en avril 1506 et Oran le 18 mai 1509 (voir Lorenzo Galindes de Carvajal, Anales breves del reinado de los reyes catolicos D.Fernando y Dona Isabel de gloriosa memoria, dans Cronica de los reys de Castilla, Madrid, D. Caetano Rosell éd., vol. III, t. LXX, 1953). Il réussit également à annexer le projet de D. Manuel, en se rapprochant des vues de Jules II, pour qui la protection de l'Italie contre le péril ottoman était plus importante que la conquête des lieux saints. Cependant, les difficultés militaires du Portugal au Maroc en 1508 expliquent également le report du plan de D. Manuel.

L'auditoire de la conférence s'est donc attaché d'abord à la lecture, à la transcriptions et au commentaire de la lettre de Frei Diogo de Montemor adressée à D. Manuel (AN/TT, Núcleo Antigo 879, no 243, datée de Messine, le 18.XII.1507, f. 1-2). En préparation de la conférence de l'année prochaine, qui portera sur le conflit lusomamelouk (1509-1512) et les réseaux d'espionnage en Méditerranée d'après la documentation de l'Archivo General de Simancas, il a semblé pertinent de commencer à exploiter les documents suivants de cette série, datant des années 1509-1512. Quatre de ces documents ont été transcrits par António Manuel Lázaro dans la thèse de doctorat mentionnée l'année dernière, mais n'ont pas été édités à ce jour. On a donc lu et commenté le document du Corpo Cronológico, II, 22, 70 (une autre copie étant conservée dans II, 22, 139), comportant la traduction d'une lettre de l'abbé de SainteCatherine du mont Sinai adressée au grand-maître de l'ordre de Saint-Jean de Jérusalem, suivie d'extraits de deux autres, la première envoyée à Jacomo do Campo, marchand demeurant à Rhodes, et la seconde de Frei André do Amaral, en provenance également de Rhodes, avec des nouvelles des Mamelouks, de la mer Rouge et des Indes (Le Caire et Rhodes, 2 et 16.VI.1510). Trois autres lettres, envoyées par André do Amaral à D. Manuel (rappelant les événements de 1501-1508) ont été également étudiées : la I, 11, 47 (Rhodes, 29.III.1512, f. 1-3v) suivie d'une autre, non datée, mais probablement de mars ou avril 1512 (f. $3 \mathrm{v}-4$ ), ainsi que la I,11,61, f. 1. 


\section{Le Portugal et l'Allemagne hitlérienne}

Comme annoncé dans le rapport de l'année 2011-2012, la conférence de cette année s'est attachée à étudier les relations culturelles entre le Portugal et l'Allemagne hitlérienne. Nous avons d'abord examiné les échanges en matière d'enseignement. On doit à Gustavo Cordeiro Ramos, professeur de germanistique à l'université de Lisbonne, ancien ministre de l'Éducation, l'introduction (le 29 septembre 1933) de la langue allemande dans les établissements d'enseignement secondaire portugais. En 1941, fut inaugurée à l'université de Lisbonne la chaire permanente en histoire de la littérature allemande, dont le premier titulaire fut Wolfgang Kaiser, un ami de l'éminent germaniste portugais Paulo Quintela (1941 à 1946). L'Institut allemand de l'université de Coimbra fut fondé le 5 août 1925 par João da Providência Sousa e Costa, avec le concours de Bernard Schädel [† 1926] (créateur de l'Institut ibero-américain de Berlin). L'Institut collabora avec le Grémio Luso-Alemão et la Deutscher Studensaal d'économie liée à l'École supérieure de commerce de Lisbonne. À l'exemple de l'Espagne, les échanges culturels avec le Portugal ont été strictement encadrés dès la fin des années 1930. Le NSDAP ( $A O$-Auslandsorganisation) à qui échut la mission d'organiser idéologiquement les ressortissants allemands de l'étranger selon une hiérarchie bien précise, envoya ses agents dans plusieurs pays grâce à la section 33 du NSDAP dirigée à l'époque par Ernst Wilhelm Bohle. En 1936, les représentations diplomatiques et consulaires à l'étranger reçoivent ainsi des cadres de la AO. Dans le cas du Portugal, l'implantation du NSDAP aurait été favorisée par l'existence du Stahlhelm, une association de combattants de la $1^{\text {re }}$ guerre mondiale fondée en 1918 par Franz Seldte. Grâce aux relations avec Friedhelm Burbach, un commerçant établi à Porto en 1923 et des membres du club allemand de Barcelone, un groupe local du Stahlhelm fut constitué en 1928. Le groupe initial du NSDAP comptait 7 personnes au $1^{\text {er }}$ janvier 1931. Entre le 15 septembre 1930 et le 31 décembre 1931 il gagna 19 membres; le chiffre monta ensuite à 40 adhérents. Pendant cette période Friedhelm Burbach assura la direction du groupe national ; la direction du groupe de Porto (Ortsgruppe) fut donnée à Hans Steinmetz. Le groupe local de Lisbonne fut crée en 1932 et dirigé par Robert Heidecke. En septembre 1933 ce même groupe était dirigé par Julius Claussen. Pour des raisons logistiques et diplomatiques, et dans la mesure où le NSDAP / Espagne avait la priorité sur celui du Portugal, la compétence de Burbach fut étendue à toute la péninsule Ibérique. En avril 1934, Claussen devint le dirigeant du groupe national Portugal du NSDAP tandis que Wolfgang Gräser assumait la direction du groupe local (il fut remplacé par Paul Wehr en 1937). Claussen décèda en 1940 et plusieurs personalités portugaises assistèrent à ses obsèques, dont le secrétaire personnel du président de la République. Ribbentrop envoya une couronne de fleurs et Rudolph Hess un télégramme de condoléances. Cependant, à la suite de Hans Thomsen, il reviendra au juriste Horst Lübbe, nommé en 1941, de développer les relations culturelles avec le Portugal. Disparu en 1944 sur le front de Russie, il fut remplacé par Werner Pankow, qui parlait très bien le portugais et connaissait fort bien le Portugal.

Les efforts du NSDAP / Portugal ont été reconnus à Berlin, car, en septembre 1942, une série de membres du groupe national (Fritz Eggert, Hans Eismannn, Josef Hell, 
Friz Schubeius, Kurt Schüppel et Ernst Strasen, dirigeant de la propagande) ont été récompensés de la croix de guerre, $2^{\mathrm{e}}$ classe sans épées. En mai 1942 Lübbe reçut cette distinction pour ses mérites dans le domaine de la propagande par le cinema. De grandes expositions portant sur le $7^{\mathrm{e}}$ Art furent ainsi organisées à Lisbonne : début octobre 1943, la « Première exposition d'Art et industrie cinématographique » ouvrait ses portes dans les locaux du casino Estoril en présence de Marika Rökk et de son mari Georg Jacoby, conviés à la présentation du film de l'actrice Der Tanz mit dem Kaiser. La musique occupa également une place privilégiée dans ces échanges. En mars 1941 vint à Lisbonne le Regensburger Domspatzen. Des spectacles eurent lieu au théâtre national de S. Carlos et à la basilique de Fátima. En avril 1942 ce fut au tour des petits chanteurs de Vienne de se produire dans la basilique de la Estrela à Lisbonne et aussi à Porto. Des orchestres de musique de chambre donnèrent des concerts au Portugal, ainsi que la chanteuse Gerda Lammers (février 1943), les pianistes Walter Gieseking, Wifrind Wolf, Edwin Fischer et le quartet Schneiderhan de Vienne (mai-juin 1944). L'orchestre philharmonique de Berlin joua son repértoire à Lisbonne et à Porto (sous la direction de Karl Böhm et de Hans Knapperbusch). En juin 1943, l'opéra d'État de Berlin présenta à Lisbonne « Tristan et Iseult ». En mai 1944, les spectacles de l'orchestre philharmonique de Berlin dans le Coliseu dos Recreios et à S. Carlos connurent un vif succès. Knapperbusch reçut la médaille de Santiago d'Espada, l'une des plus hautes distinctions du Portugal.

Vitrines de la propagande allemande, les expositions ont été particulièrement soignées par le NSDAP / Portugal. Il convient ainsi de mentionner, parmi d'autres, l'exposition sur l'effort de guerre allemand organisée à l'Instituto Superior Técnico (l'École polythechnique de Lisbonne), avec le concours de l'Instituto de Alta Cultura, du Conseil publicitaire de l'économie allemande et de l'Association des ingénieurs allemands. Elle reçut environ 11000 visiteurs.

Cependant, le travail de recherche du séminaire a porté surtout sur la grande exposition intitulée Moderne architecture allemande, inuagurée à Lisbonne le 8 novembre 1941 en présence d'Albert Speer, alors inspecteur-général de l'urbanisme du Reich (Generalbauinspektor für die Reichshauptstadt, plus tard Reichsminister für Rüstung und Kriegsproduktion). Il s'agit d'un épisode important des relations luso-allemandes, brièvement signalé par quelques publications éparses (cf. Nuno Teotónio Pereia et José Manuel Fernandes, "A Arquitectura do Fascismo em Portugal », Arquitectura, 142 [1981], p. 38-49 [p. 40]; José Augusto França, «Itinerário dos Anos 40 », Colóquio Artes, 48 [1981], p. 5-8 [p. 8] [actes du colloque A Cultura e o Fascismo em Portugal, faculté des lettres de Lisbonne, mars 1980]).

Une recherche effectuée par le maître de conférences à la Bibliothèque nationale du Portugal et dans les fonds de l'Arquivo Histórico-Diplomático du ministère des Affaires étrangères à Lisbonne (décembre-janvier 2013) a permis de réunir une bonne partie de la documentation existente sur l'exposition et la visite de Speer au Portugal. Une série de télégrammes adressés par le MAE à la légation du Portugal à Berlin, section chiffre, portant sur les visas sollicités auprès de plusieurs consulats portugais en Allemagne (21.VIII.1941, $3987 / \mathrm{n}^{\circ} 346 ; 15 . X .1941,2677 / \mathrm{n}^{0} 175 ; 22 . X .1941$, $2768 / \mathrm{n}^{\circ} 183$; 31.X.1941, 3987/no 346; 4.XI.1941, 4066/n 358 ; 29.XI.1941, $3987 / \mathrm{n}^{\circ} 346$ ) ont permis tout d'abord d'identifier les architectes et collaborateurs 
qui accompagnèrent Speer (Wolters, Renner, Bibrach, Hermann Distel, Bruggmann). L'exposition, reportée le 14 octobre 1941 en raison du torpillage du bâtiment portugais Corte Real par un sous-marin allemand, a ouvert finalement ses portes le 8 novembre 1941 à l'École des beaux-arts de Lisbonne (le vernissage était prévu pour le $1^{\text {er }}$ novembre 1941). Elle fut organisée, du côté portugais, par les soins de l'architecte Pardal Monteiro, président de la Société nationale des beaux-arts (SNBA) et par Eugénio Correia (président du syndicat national des architectes) avec la participation du Grémio Luso-Alemão. Le commissaire scientifique fut Rudolf Wolters (Richard Walters dans la presse portugaise), l'un des plus proches collaborateurs de Speer. Otto Renner et G. H. Theunissen collaborèrent en tant que commissaire-adjoint et attaché de presse respectivement.

Le maître de conférences a pu consulter à la Bibliothèque nationale du Portugal (BNP) sous la cote B.A.1430 A., un exemplaire du catalogue de 104 pages qui accompagna l'exposition (édité à Berlin par l'Editorial Volk und Reich, 1941). Il s'agit un ouvrage bilingue portugais-allemand, réalisé par les services de propagande du Reich, car l'exposition, intitulée à l'origine Neue Deutsche Baukunst, fut d'abord présentée à Berlin. Il comporte une présentation de Speer (p. 1) une longue introduction de Wolters (p. 6-21) et une série d'illustrations en noir et blanc des travaux des architectes du Reich (p. 25-103). Outre les plans et maquettes de Speer proposés pour la reconstruction de Berlin, y figurent également les œuvres de Paul Ludwig Troost, Kurt SchmiedEhmen, Ludwig et Franz Ruff, Joseph Torak, Arno Brecker, Willelm Kreis, Hugo Röttcher, Ernest Sagebiel, Herman Giesler, Paul Bonatz, Hans Freese, Fritz Tamms, Götthold Nestler, Wolfgang Binder et Paul Baumgarten.

Une recherche dans le Diário de Notícias, le grand quotidien porte-parole du régime, conservé aujourd'hui à la $\mathrm{BNP}$, a permis de mieux s'informer sur l'exposition (outre les renseignements données par le numéro 2 de la revue Signal (1941); nous avons ainsi dépouillé une dizaine d'articles échelonnées entre le 26 octobre et le 23 novembre 1941. Il ressort qu'une partie du matériel à exposer arriva (de Madrid ?) le 26 octobre, transporté dans 11 wagons. Le montage avait requis les compétences de dizaines d'ouvriers dirigés par « des artistes allemands spécialisés » (article du 2 novembre 1941). La presse fut conviée le 4 novembre, pour une visite à $17 \mathrm{~h} 30$ (articles du 4 et 6 novembre). L'article du 2 novembre, qui saluait « un événement artistique de grande portée » comportait une fiche biographique d'Albert Speer, de ses réalisations et de ses fonctions au sein du Reich. L'inauguration eut lieu le 7 novembre en présence du président de la République portugaise, le général Óscar Carmona, du chef de sa maison militaire, de ses aides de camp et secrétaires ; de Duarte Pacheco, le ministre des travaux publiques de Salazar accompagné du sous-secrétaire d'État, Espregueira Mendes ; du sous-secrétaire à l'Éducation nationale, Lopes de Almeida ; du gouverneur civil de Lisbonne, Rodrigues de Carvalho; d'António de Meneses (SNI) ; de Guerra Viana et de Sousa Lopes. Étaient aussi présents António Eça de Queiroz, directeur-adjoint du Secrétariat national de l'information (SNI), Silva Dias, directeur des services de production de la Radio nationale (Emissora Nacional), les architectes Pardal Monteiro et Eugénio Correia, le dernier étant le président de la Société des beaux-arts et du Syndicat national des architectes. Le côté allemand fut représenté par le baron von Hoyningen-Huene, chef de la légation et ministre 
d'Allemagne, accompagné par son personnel, les fonctionnaires du service consulaire et les membres de la colonie allemande. L'ambassadeur d'Espagne, les ministres de Roumanie, d'Italie, de Hongrie et du Japon, le gouverneur militaire de Lisbonne, plusieurs dizaines de journalistes, artistes, architectes et intellectuels avaient été également conviés (Mircea Eliade, arrivé à Lisbonne le 10 février 1941 pour y occuper le poste de conseiller culturel de l'ambassade de Roumanie fut certainement l'un des invités). L'architecte Raul Lino, qui avait fait ses études en Allemagne, servit d'interprète entre les Allemands et le président de la République. L'entrée de l'exposition était ornée de deux bustes, celui du général Óscar Carmona et celui d'Adolf Hitler. Déployant une scénographie très hitlérienne, l'exposition occupait trois grandes salles aux murs recouverts de drapés blancs et de velours. Le salon noble était dominé par l'aigle national-socialiste. Des tableaux, ainsi que des pièces de mobilier, égayaient l'austérité de plusieurs maquettes (article du 7 novembre1941), même si l'exposition ne rassemblait pas toutes les pièces présentées à Berlin. Un banquet fut ensuite offert par le couple von Hoyningen-Huene à la légation allemande.

Albert Speer était arrivé en voiture au Portugal le 8 novembre 1941 au matin, autrement dit le lendemain de l'inauguration de l'exposition, en provenance de Madrid, accompagné de Wolters et de Bruggmann. Il visita Évora, où il prit son déjeuner, et fut reçu à Lisbonne, au Cais do Sodré, par plusieurs personnalités de la capitale avant de prendre ses quartiers à la légation allemande (article du 8 novembre 1941).

L'exposition connut un énorme succès, dont témoignent les archives photographiques de l'époque, et fut considérée au Portugal comme la plus importante de ces années-là. En dépit du mauvais temps et de la pluie persistante elle reçut plus de 100000 personnes pendant les 14 jours d'ouverture (Institüt für Zeitgeschichte, München, Mikrofilmrolle MA-791/2, p. 5320323f Ifz). Elle renforça les influences culturelles allemandes sur le « groupe des Beaux-Arts », déjà visibles dans les années 1930. On a cité un exemple significatif portant sur les projets d'urbanisme de la capitale portugaise : le plus grand hopital public de Lisbonne, celui de Santa Maria, fut construit en 1940 sur des plans dressés en 1938 par l'architecte Hermann Distel, un proche de Speer, membre du groupe qui l'accompagna au Portugal. L'exposition Speer a également ouvert la voie à l'invitation d'autres architectes et artistes allemands qui ont exposé au Portugal pendant la guerre, à l'invitation du SNI. Elle a conduit notamment à la réalisation de l'exposition de 1942, déjà mentionnée, sur l'effort de guerre allemand.

Les travaux de l'année ont été clôturés par deux conférences supplémentaires de M. Sérgio Campos Matos, historien, professeur à l'université classique de Lisbonne, auteur de nombreux travaux qui font autorité sur le $\mathrm{XIX}^{\mathrm{e}}$ siècle portugais. Elles ont porté sur «Ibérismes, pan-hispanisme et nationalismes au Portugal et en Espagne $\left(\mathrm{XIX}^{\mathrm{e}}-\mathrm{XX}^{\mathrm{e}}\right.$ siècles) $»($ le 5 mai) et sur « Historiographies, mémoires nationales et nationalismes ibériques ( $\mathrm{XIX}^{\mathrm{e}}$ siècle $) »($ le 7 mai). 